\title{
Advances in 2D, 3D and 4D STEM Image Data Analysis
}

\author{
Lewys Jones, Peter D. Nellist
}

Department of Materials, University of Oxford, Oxford, UK

The aberration-corrected scanning transmission electron microscope (STEM) is an immensely powerful instrument delivering a variety of position-sensitive imaging and analytical signals simultaneously. In general STEM imaging data is two-dimensional (2D), with one or more imaging signals being recorded over an X-Y raster. The introduction of focus as a variable will yield three-dimensional (3D) data for these signals while the recording of a 2D ronchigram at each probe position will generate a fourdimensional (4D) data set. With the addition of each new dimension new challenges but new opportunities emerge in STEM imaging.

Identifying the information that can be extracted from recorded high-angle annular dark-field (HAADF) STEM images first requires scrutiny of the precise conditions of the recording. It is well known that the STEM's high precision and magnification can leave it vulnerable to environmental / instrumental instabilities such that microscope suite design is becoming increasingly important [1]. Many published images contain apparent image 'tears' or 'jogs' both parallel and perpendicular to the fast-scan direction such as those in Figure 1 (left). Whilst it is possible in some cases to restore such images using Fourierspace processing [2] it has been found by real-space analysis that often the experimental cause of these distortions is not purely periodic or even constant within a scan-line. Furthermore matching the realspace nature of the cause of these distortions with a real-space restoration allows for the restoration of images of samples with arbitrary scan-rotations, of grain-boundaries or even nano-particles dominated by free surface [3]. Using this method signal-noise ratio (SNR) and resolution improvements of up to $46 \%$ and $11 \%$ respectively have been achieved (Figure 1, right).

Expressing HAADF STEM images as a fraction of the total beam-current scattered to the detector, socalled 'quantitative STEM' [4], is growing in interest. Recently we have combined this probe-current normalisation with both robust peak-finding and advanced background-subtraction algorithms that enable us to segment images using Voronoi polygons [5] and integrate regions to find the absolute scattering cross-sections for every atomic column in free-standing samples such as nano-particles.

Introducing defocus as an experimental variable allows for the recording of focal-series yielding threedimensional data volumes. Using the two-dimensional object-function assumption and the probe-object convolution model for incoherent imaging we have developed a HAADF STEM focal-series algorithm for lattice resolution images. After re-registering data to account for specimen drift, analysis of the datavolume as a stack of Fourier-transforms allows any remnant aberrations in the STEM probe to be diagnosed [6]. Using this diagnosis these aberrations, including astigmatism and coma, can be compensated for and a single image reconstructed from the data volume free from these effects and with an improved SNR and resolution of $67 \%$ and $17 \%$ respectively (Figure 3).

Lastly recording the STEM detection plane with a pixilated CCD (two-dimensional) over a raster of probe positions (also 2D) yields a four-dimensional data set [7]. This offers a wealth of opportunity for effectively introducing the imaging detector in post processing. This virtual-detector can be circular, semi-circular, annular, wedge-shaped, wedge-annular etc. If an annular detector is used an annular- 
bright-field image is produce where as if the difference between two opposite segments is used a differential phase contrast (DPC) image can be generated, Figure 4 (right). In this way several image types can be extracted from the same data set and beam-dose. At present limitations of this technique include the relatively slow read-out time of the pixilated CCD and the sample drift over this time. Also when recording 4D data the data sets rapidly become very large (over $2 \mathrm{~Gb}$ for a 16x16 array of $1024 \times 1024$ detector plane images). This introduces as many data storage and manipulation challenges as the acquisition itself.

\section{References:}

[1] Muller, D. A. et al. Ultramicroscopy 106, (2006) p. 1033-40.

[2] Braidy, N. et al. Ultramicroscopy 118, (2012) p. 67-76.

[3] Jones, L. \& Nellist, P. D. Microscopy and Microanalysis Proceedings, (2012) p. 1232-1233.

[4] Lebeau, J. M. \& Stemmer, S. Ultramicroscopy 108, (2008) p. 1653-8.

[5] Rosenauer, A. et al. Ultramicroscopy 111, (2011) p. 1316-27.

[6] Loane, R. F., Xu, P. \& Silcox, J. Ultramicroscopy 40, (1992) p. 121-138.

[7] Kimoto, K. \& Ishizuka, K. Ultramicroscopy 111, (2011) p. 1111-6.

[8] The authors acknowledge Peng Wang, Armand Béché, Tim Pennycook and the EPRSC.

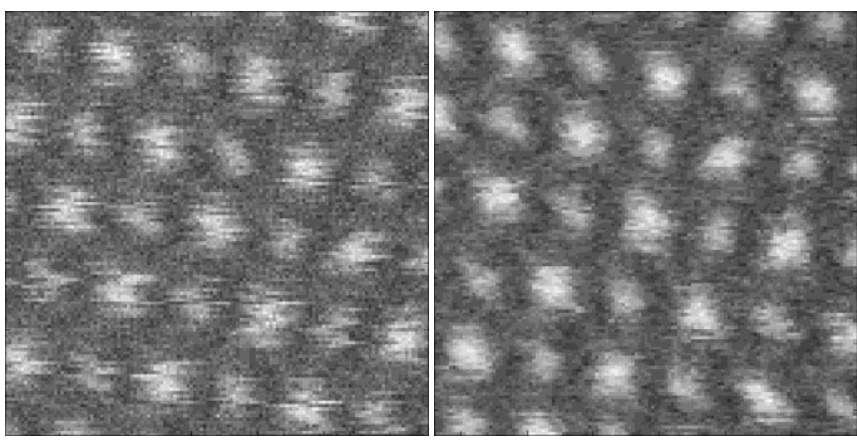

Figure 1. Enlargement from a HAADF STEM image of [100] oriented $\mathrm{SrTiO}_{3}$ before (left) and after (right) scan-noise and drift correction (SNR improved 46\%, resolution improved 11\%).
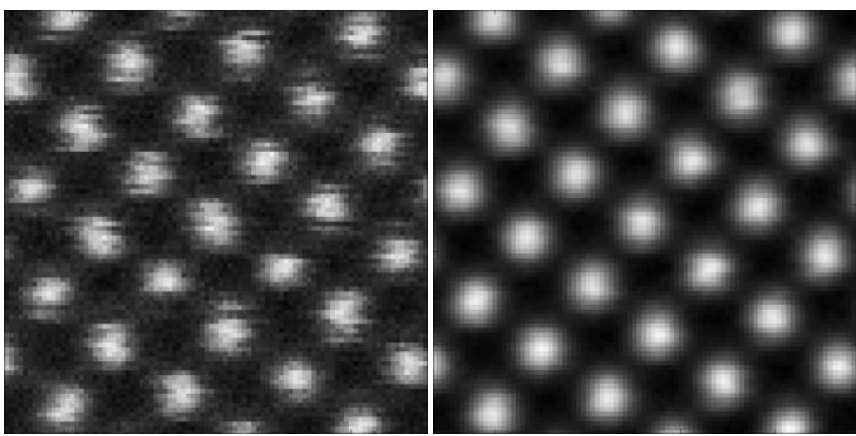

Figure 3. Enlargement from median frame of a HAADF STEM focal-series of [100] oriented $\mathrm{SrTiO}_{3}$ (left) and the corresponding restoration (SNR improved 67\%, resolution improved 17\%).

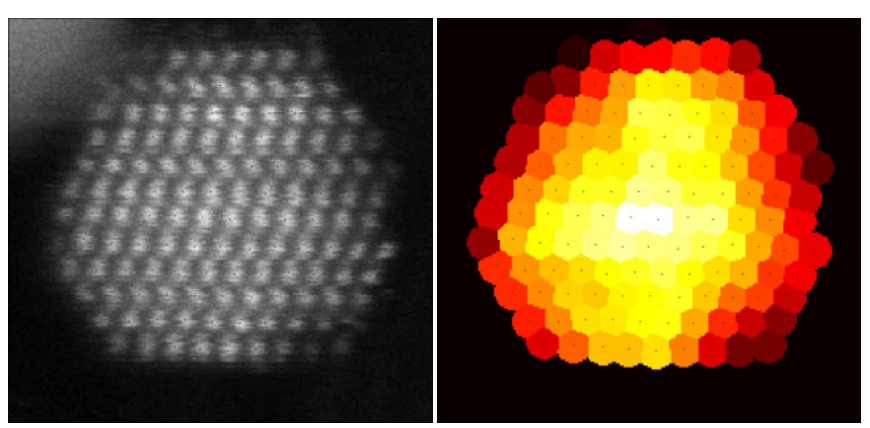

Figure 2. HAADF STEM image of a [110] oriented Pt nano-particle (left) and the corresponding quantitative scattering cross-sections map (right).

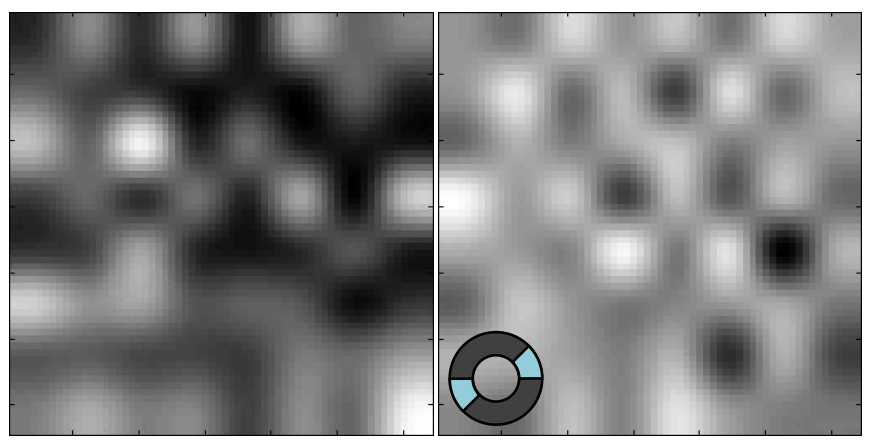

Figure 4. Matched pair of STEM HAADF (left) and extracted ABF (right) images. Inset schematic indicates the two annular difference wedges used. 